\title{
Oxidative Stress Induced by Lead in Chloroplast of Spinach
}

\author{
Xiao Wu • Hao Huang • Xiaoqing Liu • Liang Chen • \\ Chao Liu $\cdot$ Mingyu Su $\cdot$ Fashui Hong
}

Published online: 1 July 2009

(C) Humana Press Inc. 2009

\section{Erratum to: Biol Trace Elem Res (2008) 126:257-268 DOI 10.1007/s12011-008-8195-7}

The original version of this article unfortunately contained a mistake. The authors' first name and last name were interchanged in the original article. The correct names are shown above.

The online version of the original article can be found at http://dx.doi.org/10.1007/s12011-008-8195-7.

X. Wu $\cdot$ H. Huang $\cdot$ X. Liu $\cdot$ L. Chen $\cdot$ C. Liu $\cdot$ M. Su $\cdot$ F. Hong $(\bowtie)$

Medical College of Soochow University, Suzhou 215123, People's Republic of China

e-mail: Hongfsh_cn@sina.com 